\title{
Distribution of Serotypes and Antimicrobial Resistance of Streptococcus Pneumoniae in a Children's Hospital in Nepal
}

\author{
Sherchand JB' ${ }^{1}$, Joshi $\mathrm{AR}^{2}$, Gauchan $\mathrm{P}^{3}$, Amatya $\mathrm{J}^{4}$ \\ ${ }^{1}$ Prof. Jeevan Bdr Sherchan, MSc Trop.Med. PhD (U.K.); Professor, Dept of Microbiology and Chief of Health \\ Research Laboratory. ${ }^{2}$ Ashish Raj Joshi (MSc Medical Microbiology), College of Science and Technology, Kalimati, \\ ${ }^{3}$ Punita Gauchan; (MSc Medical Microbiology) Research Officer: Tribhuvan University Institute of Medicine, Health \\ Research Laboratory, Department of Clinical Microbiology, Maharajgunj, Kathmandu, Nepal, ${ }^{4} J y o t i$ Amatya; (MSc \\ Medical Microbiology), Assistant Professor in Microbiology: College of Science and Technology, Kalimati, Nepal.
}

Address for Correspondence: Prof J.B. Sherchand. E-mail: itdrc@healthnet.org.np

\begin{abstract}
Introduction: Streptococcus pneumoniae in low number is a part of normal nasopharyngeal and oropharyngeal flora of many healthy persons and also children, which generally remains harmless unless provoked by viral infections such as common cold, influenza etc. In this situation, pneumococcus is secondary pathogen but may be primary pathogen in immunocompromised people. Bacterial colonization of nasopharynx starts immediately after birth without development of disease. Objectives: The study was conducted to determine the prevalence of nasopharyngeal colonization with $S$ pneumoniae, to determine their antibiotic susceptibility pattern and to determine distribution of different serotypes. Methods: Hospital based prospective study was conducted from February 2007 to September 2007. All the specimens were inoculated into $7 \%$ sheep blood agar and incubated in $5-10 \% \mathrm{CO}_{2}$ atmosphere at $37{ }^{\circ} \mathrm{C}$ for 24 hours. $S$ pneumoniae was identified by colony morphology, Gram's stain, optochin susceptibility test and bile solubility test. Antibiotic susceptibility test was performed by modified Kirby-Bauer disc diffusion method. The in vitro susceptibility of the Oxacillin resistant isolates to Penicillin was determined by the E-test. Serotypes were determined by bacterial co-agglutination using the Pneumotest Kit (Statens Serum Institut, Denmark) with 12 pooled antisera. Results: In the study, $34.6 \%$ of the children were found to be nasopharyngeal carriers of $S$ pneumoniae among which $50.8 \%$ were male and $49.2 \%$ were female. Cephotaxime (100\%), Chloramphenicol (100\%) and Erythromycin (98.5\%) were most effective antibiotic against $S$ pneumoniae and the least effective was Cotrimoxazole (60\%). 15.4\% were found to be resistant to Oxacillin. On performing E-test of Oxacillin resistant strains against Penicillin, all were found to be susceptible to Penicillin. The isolated strains were found to belong to 16 different serotypes whereas $8 \%$ were Non-typeable. Conclusions: Carriage rate is similar both in male and female. Penicillin resistance has not arisen in $S$ pneumoniae isolated from nasopharynx which is causing problem world wide.
\end{abstract}

Key words: Children, Nasopharyngeal Swab, S pneumoniae, Serotype, E-test

\section{Introduction}

Respiratory tract infection is the major health problem in developing countries. Infection of the respiratory tract is the most frequent and important cause of short term illness in the population. It is frequently the first infection to occur after birth, and too often the final illness before death ${ }^{1}$.

Although, majority of these respiratory tract infections are viral in origin, secondary bacterial infection is also common, particularly in malnourished and very young. Resident bacteria of upper respiratory tract such as $S$ pneumoniae, $H$ influenzae, $S$ pyogenes are the most common causative agents of respiratory tract infections. $S$ pneumoniae is chiefly involved in infection of upper and lower respiratory tract. Some important respiratory tract infections that are caused by $S$ pneumoniae are sinusitis, otitis media, bronchitis, pneumonia etc ${ }^{2}$.

Approximately 2.6 million children under 5 years of age die annually of pneumonia predominantly in 
the developing world; approximately one half of these deaths are attributable to $S$ pneumoniae either solely or in conjunction with a viral respiratory infection, malnutrition or HIV infection ${ }^{3}$.

People usually carry pneumococci without symptoms, but under certain circumstances, this carriage can also contribute to respiratory or even systemic disease. Infection usually occurs in a person already colonized with $S$ pneumoniae, when the bacteria invade into the patient's body. Several factors have considerable impact on pneumococcal carriage and its rates ${ }^{4,5}$.

The bacteria $S$ pneumoniae carried in the nasopharynx of children reflect the infection causing strains currently circulating in the community. So studies of the prevalence of different pathogens and their resistance patterns can provide useful indications for more rational therapeutic and preventive strategies. The nasopharyngeal carriage of $S$ pneumoniae in young children has been related to the development of disease and the spread of the pathogen and it has been found to belong to a limited number of serotypes that are also some of the most common cause of invasive pediatric diseases ${ }^{6}$. Considering these facts, the current study was carried out.

\section{Methods}

A total of 188 children of age between 2 months and 5 years attending out patient department of Kanti Children's Hospitalwere included in the study after taking consent from guardian of a child and also from the child whenever possible. Nasopharyngeal swab specimens were collected using specifically designed pediatric sized swab of thin flexible aluminum shaft tipped with Dacron polyester (Copan Diagnostics Corona, Ca USA) and transported to Health Research Laboratory, Institute of Medicine, Tribhuvan University Teaching Hospital in Skim milk tryptone glucose glycerin (STGG). In the laboratory, the specimens were vortexed and inoculated on blood agar supplemented with $7 \%$ sheep blood and incubated at $37{ }^{\circ} \mathrm{C}$ for 24 hours in $5-10 \% \mathrm{CO}_{2}$ atmosphere. $S$ pneumoniae was identified by colony morphology, Gram's stain, optochin susceptibility test and bile solubility test. Antibiotic susceptibility test was performed by modified Kirby-Bauer disc diffusion method on Mueller-Hinton Agar supplemented with $7 \%$ sheep blood and interpreted according to National Committee for Clinical Laboratory Standards (NCCLS) recommendation. E-test of Oxacillin resistant strains of pneumococci was performed against Penicillin by using E-test strip of Penicillin (AB Biodisk North America, Inc) and interpreted according to NCCLS. Serotyping of the isolated pneumococci was done by coagglutination method using Pneumotest kit with 12 pooled antisera. It was based on capsular reaction due to interaction between pneumococcal capsular polysaccharide and its homologous antibody.

\section{Results}

Of 188 specimens investigated for $S$ pneumoniae, $65(34.6 \%)$ showed the growth of $S$ pneumoniae. Out of the 65 isolates, $33(50.8 \%)$ were male and $32(49.2 \%)$ were female (Figure 1). Gender wise distribution of nasopharyngeal carriage of $S$ pneumoniae was statistically not significant $(\mathrm{P}>0.05)$.

Fig. 1: Showing children enrolled and gender wise distribution of $S$ pneumoniae among them.

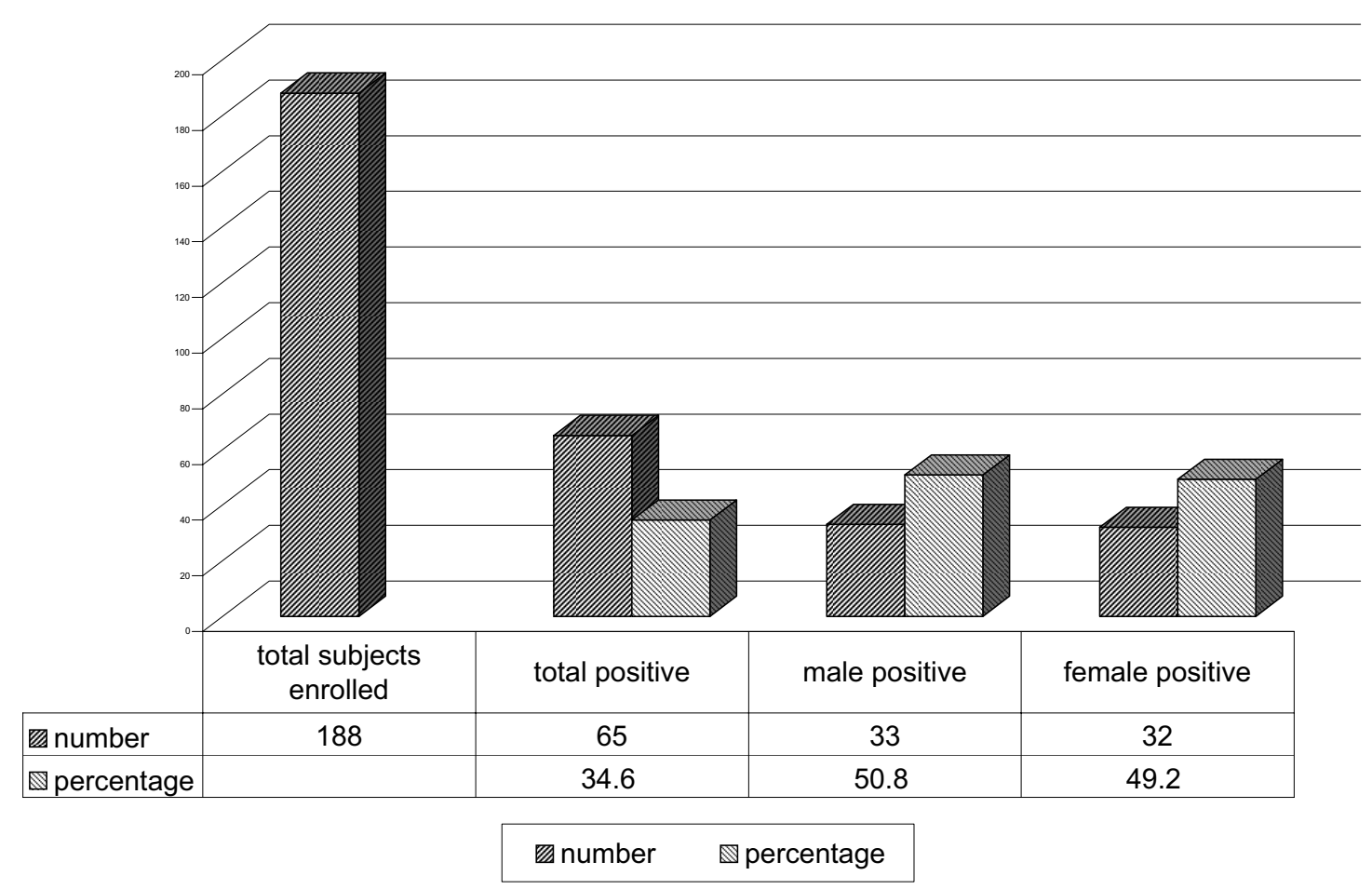


Fig. 2: Showing antibiotic susceptibility pattern of nasopharyngeal pneumococcal isolates.

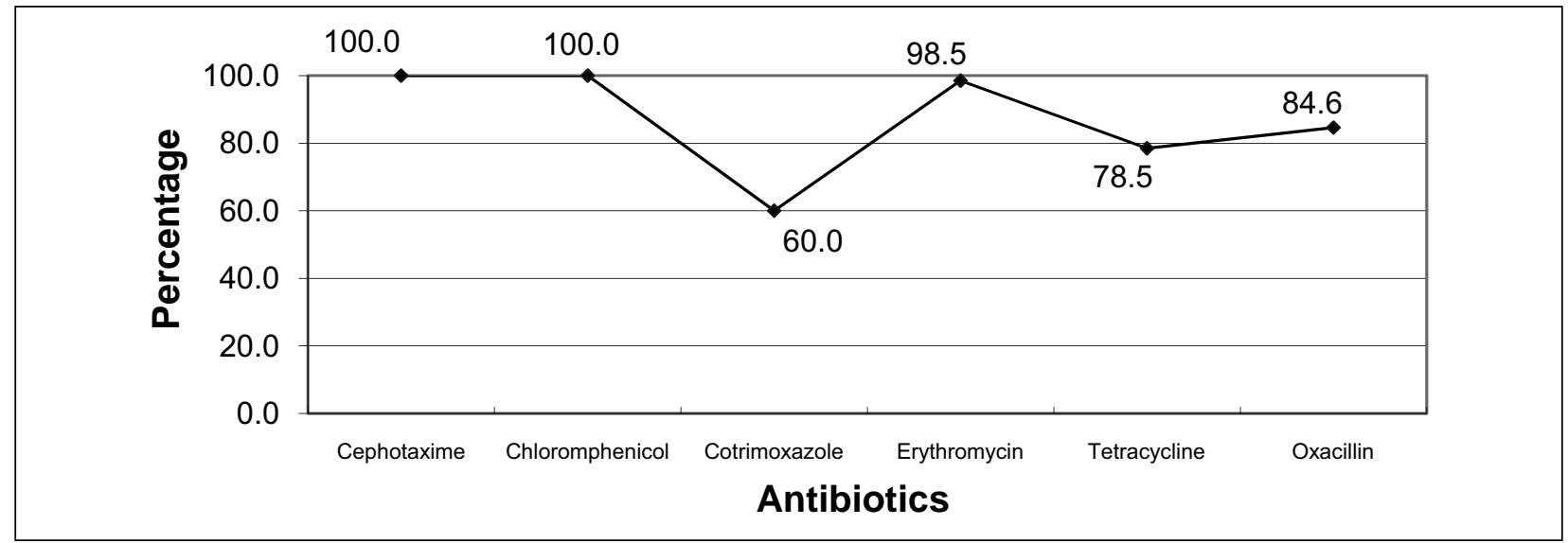

Cephotaxime, Chloramphenicol and Erythromycin were found to be most effective drugs against the isolates. Cotrimoxazole was the least effective drug. $15.4 \%$ of the isolates were found to be Oxacillin resistant (Figure 2).

On performing E-test of Oxacillin resistant strains against Penicillin, Minimum Inhibitory Concentration (MIC) of Penicillin was found to lie below $2 \mu \mathrm{g} / \mathrm{ml}$ indicating these isolates to be susceptible to Penicillin.

The isolated strains of $S$ pneumoniae were found to belong to 16 different serotypes whereas $8 \%$ of the isolates were Non-Typeable. Serotypes 19, 6, 15 and 8 were the most common (Table 1).

Table 1: Showing distribution of different serotypes of the isolated S pneumoniae.

\begin{tabular}{|c|c|c|}
\hline Serotypes & Number & Percentage \\
\hline $\mathbf{1 9}$ & 12 & $18 \%$ \\
\hline $\mathbf{6}$ & 12 & $18 \%$ \\
\hline $\mathbf{1 5}$ & 6 & $9 \%$ \\
\hline $\mathbf{1 4}$ & 5 & $8 \%$ \\
\hline $\mathbf{1 1}$ & 4 & $6 \%$ \\
\hline $\mathbf{2 0}$ & 4 & $6 \%$ \\
\hline $\mathbf{7}$ & 3 & $5 \%$ \\
\hline $\mathbf{3}$ & 3 & $5 \%$ \\
\hline $\mathbf{1 2}$ & 3 & $5 \%$ \\
\hline $\mathbf{1 8}$ & 2 & $3 \%$ \\
\hline $\mathbf{1 7}$ & 1 & $2 \%$ \\
\hline $\mathbf{1 0}$ & 1 & $2 \%$ \\
\hline $\mathbf{9}$ & 1 & $2 \%$ \\
\hline $\mathbf{8}$ & 1 & $2 \%$ \\
\hline $\mathbf{5}$ & 1 & $2 \%$ \\
\hline Total & 1 & $2 \%$ \\
\hline & 5 & $8 \%$ \\
\hline & & $100 \%$ \\
\hline
\end{tabular}

\section{Discussion}

Out of 188 nasopharyngeal swab specimen processed, $65(34.6 \%)$ of them showed growth of $S$ pneumoniae. Although a comparison of pneumococcal carriage rates between studies is difficult due to variable methodological factors, the pneumococcal carriage rate was a little bit lower in this study when compared to the study carried out by Todar, in which, nasopharyngeal colonization with pneumococci was found in $40 \%$ of the cases ${ }^{7}$.

Among 65 positive subjects, 50.8\% were male carriers and $49.2 \%$ were female carriers indicating absence of correlation between gender and nasopharyngeal colonization with pneumococci. The result can be correlated to the findings of Malla et al in which $49 \%$ were female and $51 \%$ were male carriers ${ }^{8}$.

All the isolated pneumococci were found to be susceptible to Cephotaxime and Chloramphenicol. Erythromycin was also effective drug of choice since only $1.5 \%$ were found to be resistant to it. Tetracycline could also be used for therapy since only $9.2 \%$ were resistant to it.

Cotrimioxazole showed lowest susceptibility to isolated pneumococci with $40 \%$ isolates resistant to it. In a similar study, Cotrimoxazole was the least effective drug against $S$ pneumoniae ${ }^{8}$. In addition to this, two studies in Pakistan had found Cotrimoxazole to be ineffective in one third of patients of pneumoniae and children under age of 1 year were especially susceptible to treatment failure. Cotrimoxazole was recommended by W.H.O. for treatment of infections caused by $S$ pneumoniae due to its lesser side effects, lower cost and easy availability. However, majority of $S$ pneumoniae in South Asia are now Cotrimoxazole resistant raising the question of whether W.H.O. should shift from Cotrimoxazole to more expensive drug for treatment ${ }^{9}$.

There are several possible mechanisms of development of resistance in pneumococci such as due to single point mutation, transformation (uptake and chromosomal exchange of free DNA from closely related strains or species), conjugative transposons (transfer and genetic incorporation of small segments of DNA during bacterial fusion events) etc ${ }^{10}$. 
Oxacillin, though it is not used in therapy, was used for predicting the resistance of $S$ pneumoniae against Penicillin due to its higher resistance to degradation during long term storage and $15.4 \%$ of the isolates were found to be Oxacillin resistant. Oxacillin resistance was high in the study when compared to $5.1 \%$ in study done by Malla et $a l^{8}$.

On performing E-test of Oxacillin resistant strains against Penicillin, MIC of two Oxacillin resistant pneumococcal isolates was found to be moderately susceptible and that of remaining eight was found to be susceptible to Penicillin. Thus, none of the isolates were found to be Penicillin resistant. E-test of Oxacillin resistant strains of $S$ pneumoniae was performed against Penicillin since disc testing of Oxacillin resistance for $S$ pneumoniae is not sufficient to distinguish between complete and partial resistance against Penicillin. Penicillin resistance among pneumococcal isolates in Asia has also emerged and is gradually increasing resulting treatment failure? .

Information on the regional distribution of pneumococcal serotype is essential for the development and use of appropriate pneumococcal vaccine in developing countries $^{11}$. Determining the serotype of $S$ pneumoniae is important as the vaccine production is based on the most common serotypes. The isolated $S$ pneumoniae were found to belong to 16 different serotypes which are shown in table 1 . Serotype 1 is regarded as the most invasive strain. Serotypes 1, 3, 5, 6, 14, 19 and 23 are considered comprehensive types in invasive pneumococcal infections. Except serotype 1, all serotypes considered comprehensive type in pneumococcal infections were encountered in the study. Serotypes 19, 6, 15, 23, $9,11,8,7,17,20$ and 22 are chiefly involved in nasopharyngeal colonization in children ${ }^{12}$.

\section{Conclusion}

There was found to be no correlation between nasopharyngeal carriage of $S$ pneumoniae and gender of children. Cotrimoxazole was found to be the least effective drug for treatment. MIC of Oxacillin resistant strains against Penicillin lied in susceptible and moderately susceptible region indicating absence of Penicillin resistance. Anti pneumococcal polysaccharide vaccine (23-valent) covers all the isolated serotypes of pneumococci. Protein-polysaccharide (Conjugate) vaccine suppresses nasopharyngeal carriage of $S$ pneumoniae. Thus, conjugate vaccine immunization followed by polysaccharide vaccine boosting might provide a foundation for life long protection against pneumococcal disease.

\section{Acknowledgement}

We are thankful to Dr. Chris Coles, Assistant Professor, Johns Hopkins Bloomberg School of Public Health and NNIPS family for their generous support to the study. We would also like to acknowledge staffs of Health Research Laboratory, Institute of Medicine, Tribhuvan University Teaching Hospital and Kanti Children's Hospital for their cooperation throughout the study.

\section{References}

1. Dawadi S, Rao BS, Khan GM. Pattern of antimicrobial prescription and its cost analysis in respiratory tract infection. $K U J \mathrm{Sci}$, Engi and Tec 2005 Sept; 1(1): 1-5.

2. Greenwood D, Slack RCB, Peutherer JF. Medical Microbiology, $16^{\text {th }}$ edition. Churchill Livingstone Publication, England 2003: 174-188.

3. O'brien KL, Nohynek H, The WHO pneumococcal vaccine trial carriage working group. Report from a WHO working group: standard method for detecting upper respiratory carriage of Streptococcus pneumoniae. Pediatr Infect Dis $J$ 2003 Feb; 22(2): 1-11.

4. Kaijalainen T. The identification of Streptococcus pneumoniae. National Public Health Institute Publication, Finland 2006: 15-38.

5. Streptococcus pneumoniae. Iowa Dept of Public Health Rev 2004: 1-4.

6. Marchisio P, Esposito S, Schito GC, Marchese A, Cavagna R, Principi N, the Hercules project collaborative group. Nasopharyngeal carriage of Streptococcus pneumoniae in healthy children: Implications for the use of heptavalent pneumococcal conjugate vaccine. Emerging Infect Dis J 2002 May; 8(5): 20-28.

7. TodarK.Streptococcuspneumoniae:Pneumococcal pneumonia. University of Wisconsin-Madison 2003.

8. Malla B, Sherchand JB, C Rajendra KB, Ghimire, Rijal BP. Prevalence of pneumococcal carriage in children and antimicrobial susceptibility pattern of Streptococcus pneumoniae isolates. SAARC J TB L Dis and HIV/AIDS 2005; II (2): 6-8.

9. Zaidi AKM. Resistant respiratory infections threaten developing countries. The APUA Newsletter 2003; 21(3): 1-2.

10. Schrag SJ, Beall B, Dowell S. Resistant pneumococcal infections: the burden of disease and challenges in monitoring and controlling antimicrobial resistance. World Health Organization 2001.

11. Siberry G, Brahmadathan KN, Pandian R, Lalitha MK, Steinhoff MC, John TJ. Comparison of different culture media and storage temperatures of the long term preservation of Streptococcus pneumoniae in the Tropics. Bulletin of the World Health Organization 2001; 79(1): 43-47.

12. Ozalp M, Kanra G, Gur D. Distribution of serotypes and antimicrobial resistance of Streptococcus pneumoniae in a children's hospital in Turkey. Turkey J Pediatr 2004; 46(4): 329-332. 